\title{
A Method of Measuring Protein Dry Weight in Solutions of Unknown Salt Concentration and an Application to Lipoproteins ${ }^{1}$
}

\author{
NANCY REID HARVIE ${ }^{2}$ \\ Biophysics Research Division, Institute of Science and Technology and \\ Department of Microbiology, University of Michigan, \\ Ann Arbor, Michigan 48105
}

Received July 27, 1972; accepted December 20, 1972

The dialysis bag dry weight method was developed for the measurement of dry weights of lipoproteins subfractionated on density gradients where total recovery of lipoprotein in the gradient was to be determined. Use of the conventional method for dry weight determination was precluded because of inconsistent concentration changes which would occur in the dialysis step due to differences in both the lipoprotein and salt concentration among gradient fractions. The method described consists of transfer of measured undialyzed samples into previously weighed bags followed by dialysis against water, lyophilization of the protein-bag combination and calculation of the protein dry weight as the difference between the bag weight and the total weight.

Since the method described incorporates dialysis in the assay, it is capable of giving an accurate protein dry weight measure of a non-predialyzed sample, whereas the conventional dry weight method gives an accurate value only of a previously dialyzed sample. The increase in the standard deviation of the overall dialysis bag method was shown to be less than double that of the conventional method for a sample of known salt concentration and there is no distinguishable difference in the central values obtained by the two methods.

The particular usefulness of this method for lipoprotein solutions was presented.

The determination of dry weight of a protein solution has traditionally been a method of choice for accurate measurement of concentration. This method has been done, conventionally, by measuring the samples, drying and weighing them and then subtracting the weight due to nonprotein

\footnotetext{
${ }^{1}$ This work was supported by the National Heart and Lung Institute, N.I.H. grants to J. Lawrence Oncley as well as by the Life Insurance Medical Research

${ }^{2}$ Part of this work was done during the tenure of a U.S.P.H.S. postdoctoral fellowship at Harvard University Medical School and at the University of Michigan.
} Fund. 
solute, if any, of the solution in which the protein is dissolved (1). It is inherent in this method that the concentration of the nonprotein solute must be known in order to have this necessary control.

Over the last several years the attention of this laboratory has been directed toward a study of the chemical composition of human very low density lipoproteins (VLDL) and of subfractions of the VLDL distribution generated by preparative zonal rate ultracentrifugation through linear salt gradients (2). The constituent composition, i.e., peptide, phospholipid, cholesterol, and triglyceride, of each unfractionated lipoprotein preparation and of each subfraction was determined chemically and a dry weight determination was used to check the total weight recovery of the chemical analysis and to determine the percentage each constituent was of the lipoprotein fractions.

At the outset it became apparent that the conventional dry weight method was unsuitable, as such, for dry weight measurements on salt gradient fractions in which the exact concentration of salt was unknown. Dialysis of the gradient fractions against a salt solution of known concentration prior to dry weight determination would have resulted in unknown changes in the concentration of the lipoprotein caused by solvent flow across the membrane which would not have been consistent among gradient fractions with differing protein concentrations and differing salt concentrations.

Modifications of the method of preparation of the sample for the conventional dry weight measurement were considered and rejected. Dialysis of the samples from the gradient against distilled water with quantitative recovery from the dialysis bag was considered too inaccurate due to the insolubility of lipoproteins in water. Quantitative volumetric recovery from the dialysis bag of the lipoprotein which had been dialyzed against a salt solution of known concentration was technically very difficult, potentially inaccurate, and tedious, especially when dealing with multiple samples. Determination of concentration changes, due to a dialysis step, by chemical analysis of one of the lipoprotein constituents or by light absorption suffers from the larger inherent error of these methods.

For these reasons, we adapted a dry weight method reported briefly by Lever et al. (3) which circumvents the problems discussed above. In our method the protein solution from the gradient was measured directly into a weighed dialysis bag, dialyzed against distilled water, dried, and weighed along with the dialysis bag. The final dry weight, obtained after the weight of the bag was subtracted, was that of the protein present in the measured volume transferred directly from the salt gradient. Thus, concentration changes due to solvent flow during dialysis did not affect the assay. We have modified and improved the method with regard to 
pretreatment and weighing of the dialysis bags so that reproducibility and accuracy are known and standardizable. The method is completely described here and data are presented by which certain technical aspects can be evaluated.

\section{MATERIALS AND METHODS}

A semimicrobalance, Mettler Type H 16 (Scientific Products, Evanston, IL) was used for all weight determinations.

A dry-air oven, Precision Scientific Co., Thelco, Model 17, was used for preliminary drying of weighing bottles and dialysis bags and for the drying of $\mathrm{Na}_{2} \mathrm{SO}_{4}$ samples.

A vacuum oven, National Appliance Co. (Portland, OR), Model 5810, with two outlets was used for vacuum-drying procedures. A $\mathrm{P}_{2} \mathrm{O}_{5}$ drying tube was attached to one outlet through which the air reentered the oven after drying was completed. A water trap immersed in an alcoholdry ice mixture was attached to the other oven outlet as well as to the vacuum pump.

Visking dialysis tubing, No. 8 (10 mm) (Union Carbide Corp., Chicago, IL) was used throughout this study.

All chemicals were reagent grade.

\section{Lipoprotein Samples Used for Dry Weight Determinations}

Human very low density lipoprotein $(\mathrm{VLDL})^{3}$ was isolated, purified, and subsequently subfractionated on linear $\mathrm{Na}_{2} \mathrm{SO}_{4}$ gradients as described in (1). In summary, VLDL was isolated from fresh, normal, fasting, human serum by ultracentrifugation at $10,000 \mathrm{~g}$ for $22 \mathrm{hr}$ at $2^{\circ} \mathrm{C}$. The top layer containing both chylomicrons and VLDL was concentrated by pervaporation and the chylomicrons removed by floating them through a linear $\mathrm{NaCl}$ gradient $(1-0.15 \mathrm{~m})$ for $30 \mathrm{~min}$ at $6600 \mathrm{~g}$. Chylomicron-free VLDL was washed once in $0.15 \mathrm{M} \mathrm{NaCl}\left(100,000 g, 24 \mathrm{hr}, 2^{\circ} \mathrm{C}\right)$. VLDL was dialyzed against $0.36 \mathrm{M}$ (density $=1.045 \mathrm{~g} / \mathrm{ml}$ ) $\mathrm{Na}_{2} \mathrm{SO}_{4}$ for use in subfractionation gradients and dry weight determinations.

Subfractionation of purified VLDL was accomplished by floating the sample $(0.5 \mathrm{ml}, 100 \mathrm{mg} / \mathrm{ml})$ through a $4.5-\mathrm{ml}$ linear gradient having the density range of $1.0217-1.0045 \mathrm{~g} / \mathrm{ml}$ for $2 \mathrm{hr}$ at $15,000 \mathrm{rpm}(18,000 \mathrm{~g})$ using a SW39L Spinco rotor. The use of $\mathrm{Na}_{2} \mathrm{SO}_{4}$ in the gradient was necessitated by the use of a modified sealed tube digestion (4) of samples prior to nitrogen and phosphorus determinations (2). Subfractions were collected using a tube cutter which was standardized so that samples of

\footnotetext{
${ }^{3}$ Abbreviations: VLDL, very low density lipoproteins; EDTA, ethylene diaminetetraacetic acid; $W L I$, weight loss increment.
} 
precisely known volume could be collected. All preparative ultracentrifugation was done on a Spinco Model L ultracentrifuge (Beckman Instruments, Inc., Palo Alto, CA). All lipoprotein and salt solutions contained $10 \mathrm{mg} / \mathrm{dl}$ of $\mathrm{Na}_{2} \mathrm{EDTA}^{3}$ at $\mathrm{pH} 7$.

\section{Dialysis Bag Dry Weight Mothod}

Visking, No. $8(10 \mathrm{~mm})$ dialysis tubing was washed by running distilled water through it for over $24 \mathrm{hr}$. The washed tubing was cut into 8-in. strips, tied securely at one end, and tested for holes with water. Each bag was folded in half and placed, free ends down, into a $13 \times 100-\mathrm{mm}$ test tube and allowed to dry at room temperature. After the bag was dry it was placed, free ends down, in a dry weighing bottle with the folded end of the bag extending above the cocked lid of the bottle. The bottle-bag combination was air-dried for $15-18 \mathrm{hr}$ at $70^{\circ} \mathrm{C}$ in a Thelco oven and placed in a desiccator to cool. At this stage the bag is referred to as the " $70^{\circ} \mathrm{C}$-dried bag". After cooling, the weight of the " $70^{\circ} \mathrm{C}$-dried bag" was obtained by weighing the bottle-bag combination, removing the hag, and weighing the bottle alone. The bag was placed in a $150 \times 22$-mm test tube marked with the number of the bottle in which it had been weighed. The weighing bottles were stored in a covered enamel tray until needed. Great care was exercised in handling the dry bags to avoid cracking the tubing.

Distilled water was added to the test tubes containing the dried bags so they would become pliable enough to add the sample. The sample of lipoprotein solution was added to the bag with a Class A, $2.0 \mathrm{-ml}$ volumetric pipet. Care was taken to avoid contamination of the inside top of the bag above the level at which the knot would be tied. A small amount of distilled water was used to wash down the inner walls of the bag after the sample was added and a firm knot was tied in the top of the bag.

The sample in the bag was dialyzed for $48-72 \mathrm{hr}$ at $2-4^{\circ} \mathrm{C}$ against two 10-liter changes of distilled water in a 12-liter battery jar. Thirty samples could be dialyzed simultaneously by attachment of the bags to a Plexiglas disc itself attached to an intermittent stirrer.

After dialysis, each bag was folded carefully and placed entirely inside the weighing bottle in which the bag had been originally weighed. Care was taken to avoid contamination. The lid of the weighing bottle was cocked and the bottle-bag combination was placed in pulverized dry ice for 15-20 min. The bottle-bag combination was then transferred to a special copper block, which har been precooled in dry ice, and which contained holes slightly larger than the diameter of the bottles and about one-third their height. The copper block containing the samples was transferred to a vacuum oven and the samples were lyophilized under 
vacuum for about $12 \mathrm{hr}$. The oven temperature was raised to $75^{\circ} \mathrm{C}$ and the samples were dried for an additional $24 \mathrm{hr}$. The bottles were capped, removed from the oven to a desiccator, and allowed to cool $1 \mathrm{hr}$ before the final weight was taken. Weighing bottles were handled by means of plastic-tipped tongs throughout the procedure.

Since it was not possible to vacuum dry the dialysis bags to constant weight before they were used as they became too brittle, control bags were run to determine the additional weight loss of the " $70^{\circ} \mathrm{C}$-dried bag" in the vacuum oven. The controls were treated in the same manner as the test bags except that distilled water was added instead of the protein solution. An average weight-loss increment per gram of " $70^{\circ} \mathrm{C}$-dried bag" (WLI) ${ }^{3}$ was calculated from the water controls. This correction factor was then applied to each test bag in proportion to its " $70^{\circ} \mathrm{C}$ "-dry weight. At least three, and usually more, controls were run with each oven load of dry weights.

The dry weight of the lipoprotein was calculated using the following equation:

Dry Weight Protein $=(C-B)-[(A-B)-W L I(A-B)]$,

where $A=$ weight $(\mathrm{g}) 70^{\circ} \mathrm{C}$-dried bag plus bottle before sample addition, $B=$ weight (g) weighing bottle alone,

$C=$ weight of the vacuum dried bottle, bag, and sample combined, and

$W L I=$ average weight loss increment $(\mathrm{g})$ of vacuum-dried bags per gram of " $70^{\circ} \mathrm{C}$-dried" bag, calculated from the water controls.

\section{Conventional Dry Weight Method}

Lipoprotein dry weights were determined by measuring a known volume of the sample which had been exhaustively dialyzed against $0.15 \mathrm{M}$ $\mathrm{NaCl}$, and of the dialyzate itself, into separate weighing bottles which had been previously dried and weighed. Samples were measured with Class A volumetric pipets. The samples were frozen and dried as described above for the Dialysis Bag Dry Weight Method. All determinations on lipoprotein were calculated by subtracting the average dry weight $(\mathrm{g} / \mathrm{ml})$ of the salt control solution from each replicate dry weight of the lipoprotein salt solution samples.

This conventional dry weight method was modified for determining the dry weight of $\mathrm{Na}_{2} \mathrm{SO}_{4}$ solutions. The samples were air dried in an oven at $110^{\circ} \mathrm{C}$ for $48 \mathrm{hr}$ without lyophilization. This modification was necessary because it was not possible to keep vacuum dried $\mathrm{Na}_{2} \mathrm{SO}_{4}$ contained within the weighing bottle, possibly due to static electricity, no matter how slowly the pressure was raised to atmospheric level at the end of the 
drying procedure. In theory the use of lyophilization and vacuum drying for protein solutions is to prevent, as far as possible, both oxidation and foaming of the sample. These precautions are not necessary when dealing with stable salt solutions.

\section{Statistical Treatment}

Dry weight data, collected under various experimental conditions, have been analyzed by comparing the variances of multiple determinations. The hypothesis $\left(H_{0}\right)$ that two variances were not different versus the alternative $\left(H_{i}\right)$ that one is greater than the other was tested using a one-tailed $F$ ratio test (5) of the variances $s_{a}{ }^{2} / s_{b}{ }^{2}$, where $a$ and $b$ refer to data groups 1 through 5 (Table 1). The critical region (C.R.) was defined as the probability ( $P$ ) that the ratio of variances of two groups being compared is greater than a certain value of $F_{u_{u}-k_{a}, n_{b}-k_{b}} / \alpha$ obtained from $F$ distribution tables (6), at some selected significance level $\alpha$, i.e.,

$$
\text { C.R. }=P\left(\frac{s_{a}{ }^{2}}{s h_{h}{ }^{2}}>F_{n_{a} \rightarrow k_{s}, n_{b}-k b / \alpha}\right)=\alpha
$$

Variances were calculated (Eq. 3 below) for groups of dry weight data consisting of multiple samples of lipoprotein or salt solutions not having the same mean but which were tested in replicate (7).

$$
s^{2}=\frac{\sum_{k} S S D}{(n-k)},
$$

where $s^{2}=$ unbiased variance of the data group,

$n=$ total number of dry weight determinations in the group,

$k=$ total number of samples in the group on which replicate dry weight measurements were made, and

$S S D=$ sum of the squares of the deviations of the replicate dry weight determinations from their mean, calculated for each sample of the group.

The coefficient of variation, $c_{x}$, (Table 3 ) is equal to the standard deviation $\left(s_{x}\right)$ divided by the mean $(\bar{x})$ of the observations (8). In this paper $c_{x}$ has been expressed as a percentage, by multiplying it by 100 , in order to express more clearly the measure of dispersion of the data which $c_{x}$ represents.

\section{RESULTS}

The dry weight data were divided into groups, designed in Table 1, so that comparisons could be made between the conventional and dialysis 
TABIE 1

Group Designation and Description, Variance $\left(s^{2}\right)$, Total Number of Replicate Determinations $(n)$, and Number of Samples $(k)$ of Dry Weight Data Used for Comparison of Variances by the $F$ Ratio Test

\begin{tabular}{|c|c|c|c|c|c|}
\hline Group & Designation & Description & $s^{2}$ & $n$ & $k$ \\
\hline 1 & Dialysis bag & Dialysis bag dry weights, lipoprotein & .0885 & 163 & 77 \\
\hline 2 & Conventional & $\begin{array}{l}\text { Conventional dry weights, salt and } \\
\text { lipoprotein }\end{array}$ & .0290 & 61 & 23 \\
\hline 3 & Foil-dried bags & $\begin{array}{l}\text { Dialysis bag dry weights, bags mis- } \\
\text { handled }\end{array}$ & 1.67 & 33 & 10 \\
\hline 4 & $t, T$ bags & $\begin{array}{l}\text { Dialysis bag dry weights, time, and } \\
\text { temperature effect }\end{array}$ & .00825 & 33 & 5 \\
\hline 5 & Control bags & Water control bags & .0144 & 116 & 25 \\
\hline
\end{tabular}

bag methods as well as among data from various experiments designed to find the source of variation in the dialysis-bag method. Table 1 also presents the variances, $n$ and $k$ values which were used to calculate the variance ratios and $F$ values given in Table 2 .

Data for the dialysis bag, conventional, and water control bag groups were collected over a period of $2 \frac{1}{2}$ years. Analyses of variance and $F$ tests done on subgroups of each of these groups, arranged according to time, showed that the variance of the subgroups were not significantly different from each other at the $5 \%$ significance level $(\alpha=.05)$ and, therefore, all data in each group were combined without regard to time of collection of the data.

The 163 dry weights determined in dialysis bags in this study ranged from 2.8 to $52.8 \mathrm{mg}$. Fifty-three percent of the weights were greater than

TABLE 2

Ratios of Variances $\frac{s_{a}^{2}}{s_{b}^{2}}$ and $F$ Values of Dialysis Bag and Conventional Dry

Weights and of Dry Weight Data Used to Test Sources of Variation of the Dialysis Bag Method ( $\alpha=.05$ )

\begin{tabular}{|c|c|c|c|c|}
\hline \multicolumn{3}{|c|}{ Experimental } & \multirow{3}{*}{$\frac{s_{a}^{2}}{s_{b}^{2}}$} & \multirow[b]{3}{*}{$F$} \\
\hline & \multicolumn{2}{|c|}{ Group* } & & \\
\hline Comparison & $a$ & $b$ & & \\
\hline 1 & Dialysis bag & Conventional & 3.05 & 1.62 \\
\hline 2 & Dialysis bag & Control bag & 6.14 & 1.43 \\
\hline 3 & Dialysis bag & $t, T$ bag & 10.72 & 1.76 \\
\hline 4 & Foil-dried bag & Dialysis bag & 16.72 & 2.04 \\
\hline
\end{tabular}

* See Table 1. 
$20 \mathrm{mg}$ and $17 \%$ were less than $10 \mathrm{mg}$. Preliminary calculations showed that the standard deviation of the dialysis bag dry weights did not vary significantly with the amount of material being weighed, probably because the major sources of variation, such as volumetric measurement and baghandling procedures, were independent of the amount of material being weighed. An expected percentage coefficient of variation $\left(c_{x} \%\right)$ can be estimated at any given weight level using the standard deviation of the method. It is apparent that both the dialysis bag and conventional dry weight methods, using the technique and equipment described, are macro methods and that samples containing $10 \mathrm{mg}$ or more of protein are more appropriate than samples of lesser amount.

The variability of the dialysis bag dry weight method is inherently greater than that of the conventional method (Table 1), because of inclusion of the bags and additional experimental manipulation in the former method. The extent of the difference in variability between the two methods is expressed by the variance ratio of 3.05 (Table 2, comparison 1 ). This ratio is greater than the $F$ value of 1.62 , and, therefore, the two variances are significantly different from each other at the chosen $5 \%$ significance level $(\alpha=0.05)$. However, the standard deviation $(0.3)$ of dry weights done by the dialysis bag method using protein solutions of unknown salt concentration is less than twice the standard deviation (0.17) of dry weights of protein solutions of known salt concentration done by the conventional method. Variability of this degree is quite acceptable for the use to which the method was put.

Three factors which might have contributed to the increased variation of the dialysis bag method were amenable to experimental analysis, namely; the variation in the weight loss increment (WLI) of the water control bags, the variation in the dry weights due to insufficient time and temperature of drying, and the variation due to mishandling of the bags during the procedure.

The variability in the weight loss increment $(W L I)$ of the water control bags was very small compared to the overall variability of the dialysis bag method (Table 1) as shown by the variance ratio of 6.14 (Table 2 , comparison 2). This ratio is greater than the $F$ value of 1.43 and thus the variances are significantly different from each other at the $5 \%$ level $(\alpha=0.05)$.

In order to test the effect of time and temperature of drying on the variability of dialysis bag dry weights, samples were dried for varying time intervals up to $96 \mathrm{hr}$ at $75^{\circ} \mathrm{C}$ and then up to $96 \mathrm{hr}$ at $85^{\circ} \mathrm{C}$. Comparing the variances (Table 1 ) of the over-all dialysis bag dry weight data and these data gave a variance ratio of 10.72 (Table 2 , comparison 3) which indicates that the variability of the dry weights in the 
dialysis bag method is not primarily a function of time and temperature of drying. Additionally it was found that there was no consistent decrease in the dry weight of the samples as the time and temperature of heating was increased.

On the other hand, preliminary drying of the dialysis bags on a flat piece of foil prior to the first weighing instead of in individual tubes, which was tried as a time economy measure, resulted in a greatly increased variance (Table 1 ) in the final dry weights. The ratio of the variances (16.72) of the over-all dialysis bag dry weight data and these data was much greater than would be expected by chance where the $F$ value would be 2.04 (Table 2, comparison 4). The necessity of careful handling of the bags for successful application of this method is emphasized by these results.

The efficiency of the dialysis of the gradient subfractions of VLDL in removing the $\mathrm{Na}_{2} \mathrm{SO}_{4}$ from the gradients was tested many times using various concentrations of $\mathrm{Na}_{2} \mathrm{SO}_{4}$. Salt controls, when dialysis was complete, became comparable to water control bags. The values of the salt controls which were run at various times throughout this study fell within the range of the water control bags with regard to variability, and dialysis was considered to be complete after a $48-\mathrm{hr}$ period with two 10-liter bath changes.

It was of interest to investigate if there was systemic variation in the dialysis bag method compared to the conventional method. A lipoprotein solution was predialyzed against a salt solution of known concentration. Samples of the dialyzed preparation were transferred directly to weighing bottles and the dry weight of the lipoprotein was determined by the conventional method which included subtraction of the weight of salt solution controls as described in the Methods section. Other samples of the same predialyzed lipoprotein solution were transferred to weighed dialysis bags and the dry weight of the lipoprotein determined by the dialysis bag method of this paper. The results are given in Table 3 . The measure of dispersion of the replicate data $\left(c_{x} \%\right)$ was greater for the dialysis bag (1.2) than for the conventional method (0.7) as would be expected from the standard deviations of the two methods. The fact that the mean value of the weights for each method were practically the same attests to the lack of systematic variation and to the accuracy of the dialysis bag method.

Total weight recovery from the gradient was calculated for 11 gradient subfractionated VLDL preparations. This calculation was made by multiplying the dry weight $(\mathrm{mg} / \mathrm{ml})$ of each gradient cut by the volume of the cut, summing these values for all cuts and dividing by the total weight 
TABLE 3

Comparison of Dry Weights and Percent Coefficient of Variation $\left(c_{x} \%\right)$ of a Single Lipoprotein (VLDL) Solution Measured by Both Dialysis Bag and Conventional Methods

\begin{tabular}{ccc}
\hline & \multicolumn{2}{c}{ Weight $(\mathrm{mg})$} \\
\cline { 2 - 3 } $\begin{array}{c}\text { Replicate } \\
\text { number }\end{array}$ & $\begin{array}{c}\text { Dialysis bag } \\
\text { method }\end{array}$ & $\begin{array}{c}\text { Conventional } \\
\text { method }\end{array}$ \\
\hline 1 & 24.28 & 23.84 \\
2 & 23.72 & 24.07 \\
3 & 23.89 & 23.95 \\
Statistics & 23.96 & 23.95 \\
$\bar{x}$ & .30 & .17 \\
$s^{a}$ & 1.2 & .7 \\
$c_{x} \%$ & & \\
\hline
\end{tabular}

a From $s^{2}$, Table 1.

of VLDL originally placed in the gradient. This latter value was the weight of lipoprotein present in $0.5 \mathrm{ml}$ of the unfractionated VLDL. For 11 subfractionated preparations the mean weight recovery was $101.4 \%$ with a standard deviation of $2.2 \%$.

\section{DISCUSSION}

The dialysis bag dry weight method presented in this paper has the striking advantage of being inherently independent of concentration changes of nondialyzable solute due to solvent flow which occurs during dialysis because the sample on which the dry weight is determined is measured before the dialysis step in contrast to the conventional method. It is well suited to experimental procedures in which complete weight recovery data of nondialyzable material is needed as, for example, in material isolated from density gradients. Although the variability of this method is somewhat greater than that of the conventional dry weight method, as would be expected, it is comparable to or less than that considered to be inherent in photocolorimetric methods. used in chemical analysis. The variability of the dialysis bag method is well within the limits required for our investigation of the chemical composition of lipoprotein subfractions, which was the use our laboratory has made of the method (2).

Comparison of average dry weights done by both the conventional and dialysis bag method, using samples predialyzed against salt solution of known concentration, gave the same mean value for replicate weights for 
both methods, substantiating both the accuracy and the lack of systematic variation in the dialysis bag method.

The weight recovery throughout the gradients, where possible errors are more than in any single measurement, showed a mean recovery of $101.4 \%$ with a standard deviation of $2.2 \%$ which is close to the variability of the dialysis bag weight measurements. This precision is more than adequate for most analytical comparisons.

The dialysis bag method had another, particular, advantage in our studies of lipoprotein subfractions, as it permitted the use of $\mathrm{Na}_{2} \mathrm{SO}_{4}$ rather than $\mathrm{NaCl}$ in the salt gradients. As pointed out in the Methods section, $\mathrm{Na}_{2} \mathrm{SO}_{4}$ is extremely difficult to handle in open containers as is required in the conventional method.

The dialysis bag method has certain disadvantages in that it is somewhat more complicated initially and somewhat more variable than the conventional method. The exact sources of the increased variability as compared to the conventional method have not been found. The factors which were tested, such as additional variable weight loss of the bags in the vacuum oven, and the time and temperature of drying were not primarily responsible in that variability due to these factors was so much less than the overall variability of the method. Such factors as contamination of either bags or weighing bottles during the steps of handling, and the possibility of mishandling the dry bags which might lead to small cracks may have contributed to the variation found, but this can, with careful technique, be minimized.

The dialysis bag method should be applicable to lipoproteins fractionated by successive ultracentrifugal flotation in solutions in which neither the dialyzable nor the nondialyzable solute concentration is known, as well as to proteins fractionated in preformed salt gradients. It should be useful for dry weight measurement on any water insoluble protein or nondialyzable material present in solutions of unknown concentration of dialyzable solute in which the concentration of nondialyzable solute, unchanged by a dialysis procedure, is desired. It is particularly useful when the dialyzable and/or nondialyzable solute concentrations are high so that dialysis previous to sample measurements in the conventional dry weight method, would potentially result in large changes in the original concentration of the material due to solvent flow. It is especially useful for lipoproteins in which it is very difficult to measure changes in concentration, due to dialysis, by either chemical or spectrophotometric measurements.

In general, this method appears to be highly satisfactory for total weight determinations in situations where the conventional dry weight procedure is inapplicable. 


\section{ACKNOWLEDGMENTS}

The author thanks Professor J. Lawrence Oncley, Associate Professors Margaret J. Hunter and Gordon L. Nordby and Dr. Walter Scheider for their excellent advice and criticism during the term of this investigation. Grateful acknowledgment is made to Miss Beverly Bazler and Mr. Fred Tung for technical assistance during part of this work.

\section{REFERENCES}

1. Hunter, M. J. (1966) J. Phÿs. Chem. 70, 3285.

2. Harvie, N. R., AND ONCLeY, J. L., in preparation.

3. Lever, W. F., Gurd, F. R. N., Uroma, E., Brown, R. K., Barnes, B. A., Schmid, K., and Schultz, E. L. (1951) J. Clin. Invest. 30, 99.

4. White, L. M., ANu Long, M. G. (1951) Anal. Chem. 23, 363.

5. Bowker, A. H., ANd Lieberman, G. J. (1955) in "Handbook of Industrial Statistics," p. 852, Prentice-Hall, Englewood Cliffs, NJ.

6. Bowker, A. H., and Liebermax, G. J. (1955) in "Handbook of Industrial Statistics," pp. 876-877, Prentice-Hall, Englewood Cliffs, NJ.

7. Hatd, A. (1952) in "Statistical Theory with Engineering Applications," p. 426, Wiley, New York.

8. HALD, A. (1952) in "Statistical Theory with Engineering Applications," p. 77, Wiley, New York. 